\title{
Overflow disrupts the genome
}

Several reports suggest a link between high glucose levels in patients with diabetes and increased risk of pancreatic ductal adenocarcinoma (PDAC). PDAC is driven by genetic alterations, with mutations in KRAS occurring most frequently and early in the disease. $\mathrm{Hu}$ et al. show that high glucose levels lead to increased glycosylation events in pancreatic cells, causing genomic instability.

To begin with, the authors analysed samples from patients with PDAC with or without prior diagnosis of diabetes mellitus (DM) and found increased levels of DNA damage in pancreatic, tumour-free tissue sections in patients with DM. Similarly, mice that received a high-sugar, high-fat (HS/HF) diet and developed high blood glucose levels had increased levels of DNA damage in pancreatic tissue sections compared with mice that were on the normal chow diet. These changes preferentially occurred in pancreatic cells - DNA damage was not observed in intestinal tissues of patients with DM or HS/HF-fed mice. Also, high glucose levels ( $11 \mathrm{mM}$ or $22 \mathrm{mM}$ ) induced DNA damage in human non-tumorigenic pancreatic cells (HPDE and HPNE cells), while the same cells grown in normal glucose levels $(5.5 \mathrm{mM})$ or other cell types (mammary, colonic or lung epithelial cell lines) grown in high or normal glucose levels were not affected.

Next-generation sequencing of $K R A S$ in pancreatic or intestinal tissues of patients with PDAC with or without DM showed that single nucleotide variations in KRAS in pancreatic tissues of patients with DM were significantly higher than in all other tissues tested. These findings were replicated in HS/HF-fed mice as well as in high glucose-treated cell lines, linking high glucose levels to increased mutations in KRAS. Using a $K R A S^{\mathrm{G} 12 \mathrm{D}}$-specific antibody, the authors identified that the number of cells containing this mutation, which is frequent in patients with PDAC, was increased in high glucose-treated HPDE and HPNE cells compared with normal glucose-treated cells.
Moreover, these cells exhibited increased anchorage-independent growth as a sign of oncogenic transformation.

In search of the mechanistic link between high glucose and DNA damage, the authors found that total levels of deoxyribonucleotide triphosphates (dNTPs), imbalance of which can induce replication stress, were reduced in high glucose-treated HPDE and HPNE cells compared with normal glucose-treated cells, and restoration of the levels of dNTPs reduced high glucose-induced DNA damage.

Glucose-derived metabolites mainly fuel energy and biomass production, but they also provide substrates for the hexosamine biosynthetic pathway (HBP). The HBP converts glucose-derived fructose6-phosphate (F6P) into uridine diphosphate- $N$-acetylglucosamine (UDP-GlcNAc), which is used for $\mathrm{O}$-GlcNAcylation of proteins, thereby regulating their activity. High glucose increased the levels of UDP-GlcNAc as well as global protein $O$-GlcNAcylation in pancreatic cells, whereas other non-pancreatic cell types were not affected. Levels of global protein $O$-GlcNAcylation were also increased in tumour-free

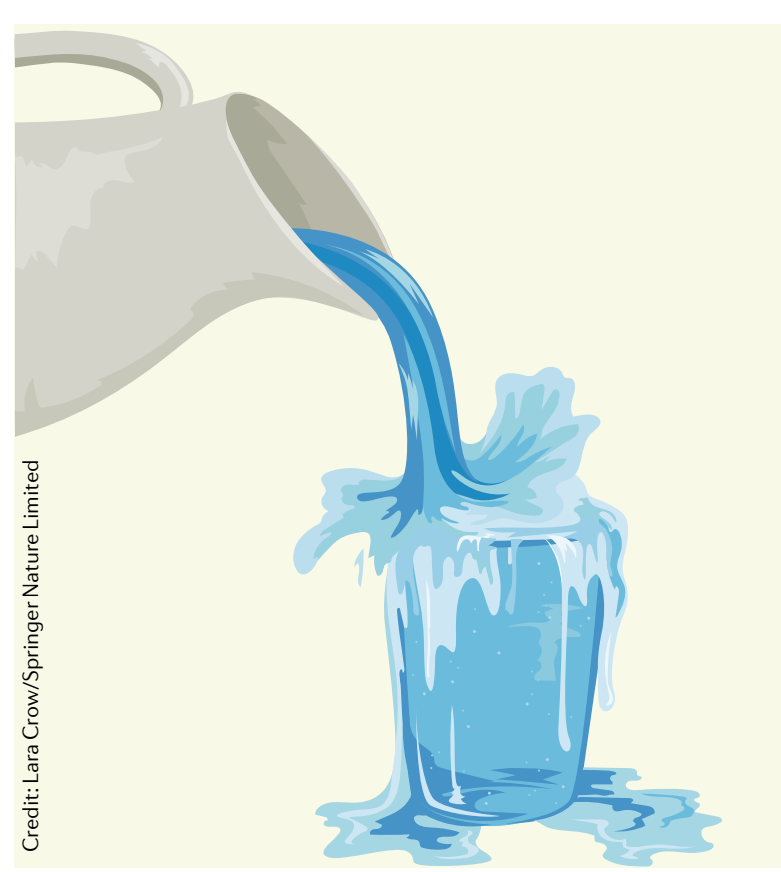

pancreatic tissue sections of patients with PDAC and DM compared with those without DM. When the enzyme responsible for this modification, $O$-glycosyltransferase (OGT), was depleted by short hairpin RNA (shRNA), levels of dNTPs were restored and DNA damage as well as anchorage-independent growth were reduced in high glucose-treated HPDE cells. Similar effects were observed when cells were treated with alloxan, a uracil analogue and reported OGT inhibitor.

To determine whether $O$-GlcNAcylation was responsible for the observed decrease in dNTP levels, the authors analysed the activity of two key enzymes involved in dNTP synthesis. Of those, only ribonucleotide reductase (RNR) was less active in high glucose-treated HPDE and HPNE cells than in normal glucose-treated cells. The authors note that RRM1, one of two RNR subunits, was hyper-OGlcNAcylated in high glucose-treated cells, leading to disruption of the RNR complex and genomic instability.

Last, the authors looked into what distinguished pancreatic cells from other cell types in their sensitivity to high glucose levels. Among glycolytic enzymes upstream of the HBP, only the activity and expression of phosphofructokinase (PFK), which converts F6P into fructose-1,6-bisphospate, were lower in high glucose-treated pancreatic cells than in mammary epithelial cells (MCF10A) or normal glucose-treated pancreatic cells. Overexpression of the major PFK isoform PFKL in pancreatic cells prevented high glucose-induced effects, whereas depletion of PFKL in MCF10A cells conferred high glucose-induced effects, indicating that low pancreatic PFK levels restrict the capacity for glycolytic conversion of glucose and cause diversion of excess glucose into the HBP.

These results require further in vivo validation but provide useful mechanistic insight into high glucose-induced oncogenic transformation in the pancreas.

Ulrike Harjes

ORIGINAL ARTICLE Hu, C.-M. et al. High glucose triggers nucleotide imbalance through O-GlcNAcylation of key enzymes and induces KRAS mutation in pancreatic cells. Cell Metab. https://doi.org/10.1016/j.cmet.2019.02.005 (2019) 